livraisons

d'Histoire

de l'Architecture

\section{Livraisons de l'histoire de l'architecture}

$26 \mid 2013$

Les ministres et les arts

\title{
La genèse de l'opéra le Peletier : un opéra provisoire pour le XIXe siècle
}

The Genesis of the Opera Le Peletier: a temporary opera for the $19^{\text {th }}$ century

Die Begründung der Oper Le Peletier: eine provisorische Oper des 19.

Jahrhunderts

\section{Elsa Cherbuy}

\section{OpenEdition}

\section{Journals}

Édition électronique

URL : http://journals.openedition.org/lha/345

DOI : $10.4000 /$ /ha. 345

ISSN : 1960-5994

\section{Éditeur}

Association Livraisons d'histoire de l'architecture - LHA

\section{Édition imprimée}

Date de publication : 10 décembre 2013

Pagination : 157-173

ISSN : 1627-4970

\section{Référence électronique}

Elsa Cherbuy, «La genèse de l'opéra le Peletier : un opéra provisoire pour le XIXe siècle », Livraisons de I'histoire de l'architecture [En ligne], 26 | 2013, mis en ligne le 10 décembre 2015, consulté le 01 mai 2019. URL : http://journals.openedition.org//ha/345 ; DOI : 10.4000//ha.345 


\section{LA GENESE DE L'OPÉRA LE PELETIER : UN OPÉRA PROVISOIRE POUR LE XIX ${ }^{e}$ SIĖCLE}

En poignardant le duc de Berry à la sortie de l'Opéra le 13 février 1820, Pierre Louvel n'atteignit pas pleinement son objectif. S'il réussit à tuer l'un des héritiers directs de la couronne, il ne mit pas pour autant un terme à cette branche fertile des Bourbons car le fils du comte d'Artois laissait son épouse enceinte d'un garçon. En revanche, le geste de l'ouvrier eut une conséquence bien plus inattendue : il mit fin à la présence de la troupe de l'Opéra rue de Richelieu. Celle-ci était installée depuis 1793 dans ce théâtre, construit par l'architecte Victor Louis pour Marguerite Montansier (ill. 1). L'entourage ultra royaliste du père du défunt, le futur Charles X, fit pression sur le roi pour condamner l'Opéra : le sang d'un enfant de France y ayant été versé, il ne pouvait décemment plus accueillir aucune réjouissance. Il fut donc décidé, malgré le parfait état de fonctionnement de la salle, qu'elle serait fermée et rasée, et qu'un monument expiatoire serait bâti à son emplacement ${ }^{1}$. Louis XVIII prit ces décisions sans avoir de recours durable pour reloger la troupe de l'Opéra, qui fut forcée de déménager au Théâtre Favart dans des conditions précaires $^{2}$. Aucune salle parisienne n'étant de taille à accueillir l'Opéra, il fallait en construire une sur mesure. Mais les conditions financières n'étaient pas réunies pour permettre au gouvernement de se lancer dans l'édification d'une salle définitive, forcément très onéreuse ${ }^{3}$. Le roi choisit une solution provisoire : un théâtre temporaire serait bâti à peu de frais, et un concours serait organisé ultérieurement pour élire un projet de salle définitive.

C'est sur cette période de prise de décision puis de construction, qui s'étale sur un peu plus d'un an, que se concentre ici le discours. L'objectif est de voir comment un monument marquant du XIX $\mathrm{X}^{\mathrm{e}}$ siècle parisien, aujourd'hui oublié, a été le fruit d'une politique précipitée mêlée de pragmatisme et d'ambition technique. J'aborderai tout d'abord les choix déterminants que dut faire l'administration de Louis XVIII : ceux de la conception de la salle, du terrain à bâtir, et enfin du nouvel architecte de l'Opéra. Les douze mois qui s'écoulèrent ensuite furent ceux

1. Une chapelle dédiée à la mémoire du duc de Berry fut effectivement bâtie à la place de l'ancien Thêatre des Arts, mais la révolution de 1830 arrêta les travaux. Le nouveau régime refusant de les financer, le monument fut finalement détruit et laissa place à l'actuel square Louvois.

2. Le Thêatre Favart était alors une propriété privée, qu'il fallut louer pour l'Opéra. Ce fut en 1825 que la Maison du roi acheta le thêâtre sur les deniers de la liste civile pour y installer le Théâtre italien.

3. Le budget de l'État était alors grevé par les indemnités de guerre dues aux alliés suite aux défaites napoléoniennes. 


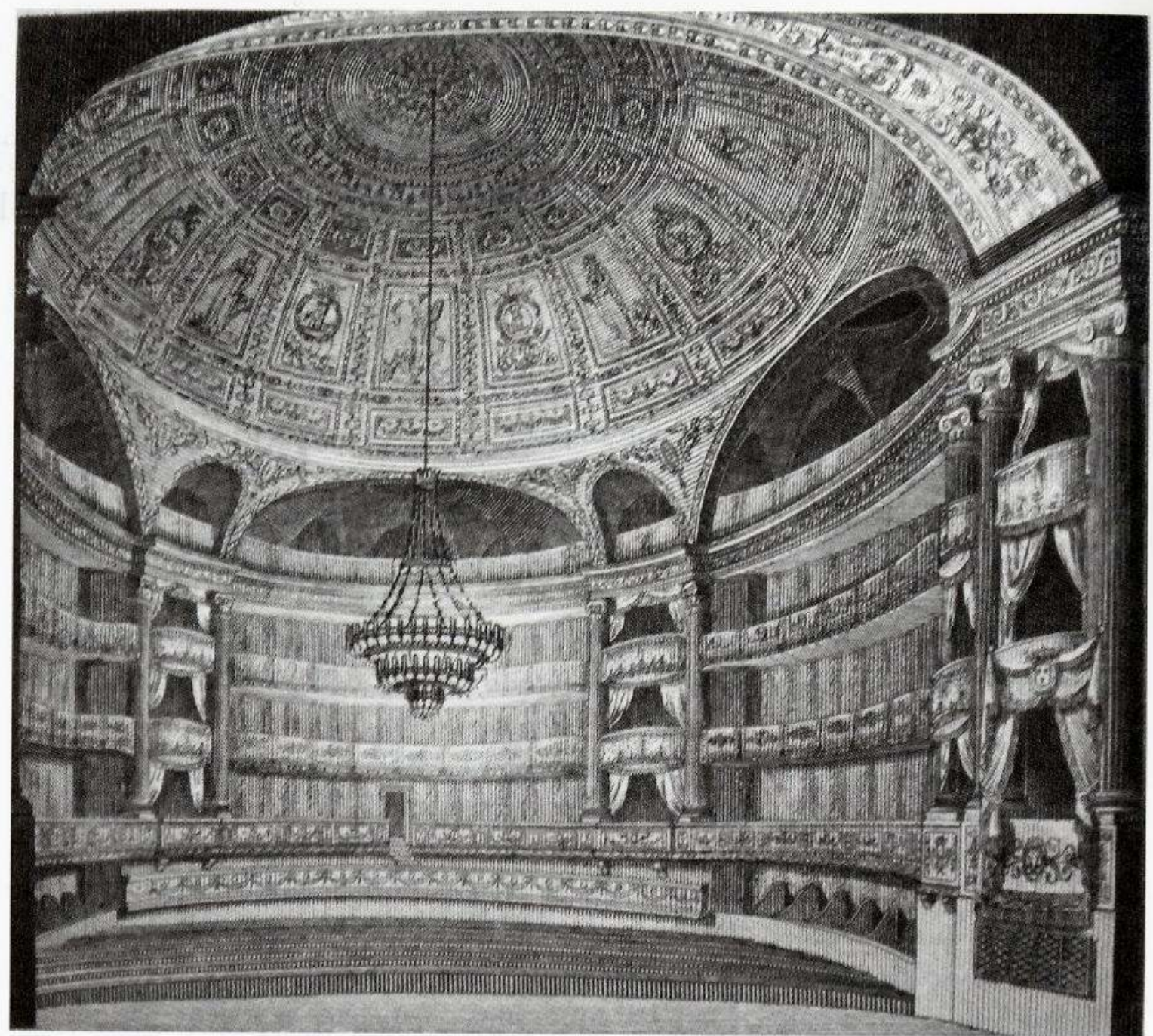

Ill. 1: Orgiazzi, Vue intérieure du Théatre des Arts, détail de la planche 13 d'Architectonographie des théâtres, 1837, BnF. Cl. de l'auteur.

des travaux de l'Opéra Le Peletier, qui s'effectuèrent dans des conditions économiques, climatiques et techniques extrêmement rigoureuses. Enfin, la livraison et l'inauguration de la salle, en retard sur les prévisions, marquèrent le début d'un demi-siècle de danse et de musique dans ce nouveau quartier de Paris, qui devint bien vite l'un des cœurs battants de la capitale.

\section{Des décisions fondatrices}

Rapidité et économie étant les maitres mots de la réalisation de la salle provisoire, il fut décidé de réutiliser au maximum les matériaux provenant de la salle de la rue de Richelieu. L'architecte alors chargé de l'entretien de l'Opéra, FrançoisJacques Delannoy (1755-1835) semble être à l'origine de cette idée. Il en fit part au comte Pradel, ministre de la maison du roi, le 18 juin 1820 :

Depuis l'événement horrible du 13 février, je me suis livré à l'étude d'une nouvelle salle d'Opéra, et [...] j’ai eu la pensée d'ériger une salle provisoire 
d'Opéra dont les dimensions seraient semblables à celles de la salle abandonnée, afin d'y transporter tout ce qui compose cette salle et son théâtre, ce qui présente une grande économie et une grande rapidité dans l'exécution de la nouvelle salle d'Opéra ${ }^{4}$.

Décision fut donc prise de démonter la salle de Victor Louis, théâtre et machinerie compris, pour la remonter à l'identique sur un nouvel emplacement. Ce qui n'aurait pas été utilisé serait vendu au poids.

Parallèlement à la réflexion engagée sur la conception de la salle, une commission fut nommée pour décider de la localisation de l'Opéra provisoire. Sa composition tenait plus d'un conseil des ministres que d'une commission des Beaux-Arts : Pierre-François-Léonard Fontaine (1762-1853) en était le seul architecte, entouré du ministre de la maison du roi, Pradel, du ministre des finances, Roy, et du ministre de l'intérieur, Siméon. Ce dernier était accompagné du directeur de la police, Mounier, et du directeur des Travaux de Paris, Hély d'Oissel. Les terrains examinés par la commission, sur l'initiative des nombreux projets proposés par des architectes volontaires, furent ceux de l'ancien couvent des Feuillants, du Palais de la Bourse, du jardin de l'hôtel de la Chancellerie place Vendôme, des jardins de l'hôtel de Wagram, de la caserne des pompiers rue de la Paix, des cours et jardins de la Bibliothèque Royale, de la place du Carrousel, de l'ancien cirque Franconi, rue du Mont Thabor, et du marché Saint-Honoré. Toutes ces propositions furent rejetées l'une après l'autre car les terrains étaient considérés comme trop petits (Feuillants, caserne des pompiers), mal adaptés (l'ancien cirque) ou impossibles à réquisitionner (marché Saint-Honoré, Bibliothèque royale, Palais de la Bourse). C'est un jeune architecte, Guerchy, qui donna l'idée de l'hôtel de Choiseul à la commission. Construit en 1743 par Antoine Matthieu le Carpentier (1709-1773) pour le financier Étienne-Michel Bouret (1709-1777), l'hôtel particulier fut l'une des plus belles propriétés du faubourg de la Grange-Batelière, alors en dehors de Paris. Il formait l'angle entre ce que l'on appelait encore la continuation de la rue Richelieu au-delà du boulevard et le cul-de-sac de la Grange-Batelière (ill. 2). Le duc de Choiseul, ancien ministre de Louis XV, l'acquit en 1780, l'agrandit en 1782 d'un hôtel voisin construit en 1716 pour Nicolas Delaunay, et laissa son nom à l'ensemble. Déclaré bien national en 1793, l'hôtel fut par la suite occupé par plusieurs administrations militaires. Cette demeure, jadis luxueuse, présentait en 1820 tous les avantages pour être choisie par la commission : elle possédait un vaste jardin permettant la construction d'un théâtre ex-nihilo, et pouvait être elle-même conservée afin d'y installer les locaux annexes de l'Opéra. Surtout, le terrain était déjà propriété de l'État, de sorte qu'il n'y avait rien à débourser pour y placer la salle provisoire. Enfin, l'état-major de la Garde nationale, qui y était établi depuis 1814, était sur le point de déménager. L'Opéra pouvait donc s'installer dans le pâté

4. Delannoy à Pradel, 18 juin 1820 , Arch. nat, $\mathrm{O}^{3} 1652$. Il reprend ici une idée déjà développée dans un de ses précédents rapports, daté du 20 mai. 


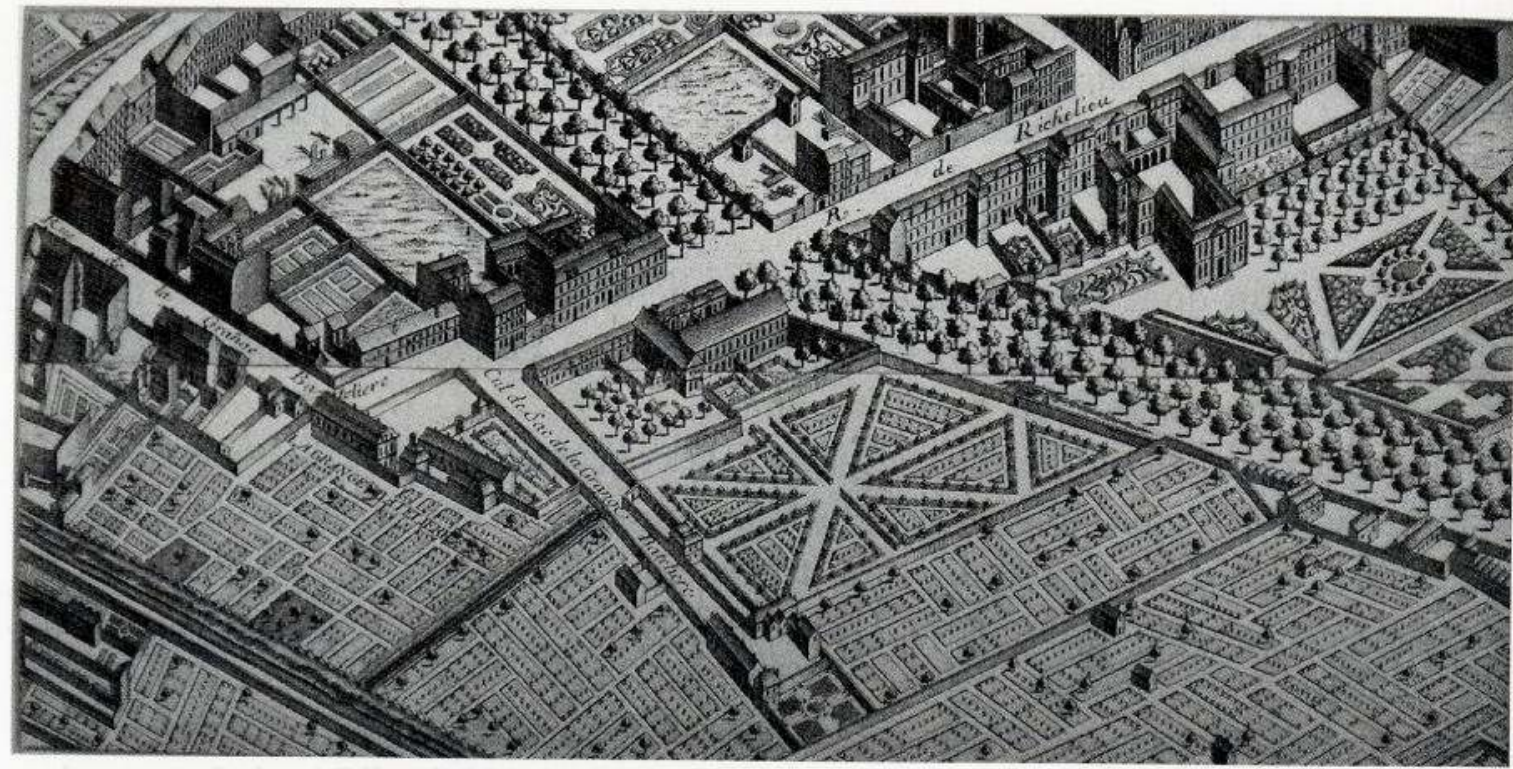

IIl. 2 : Louis Bretez, Plan du quartier de la Grange-Batelière en 1734, 1734-1736, eau-forte, Cabinet des arts graphiques du musée Carnavalet. $\mathrm{Cl}$. de l'auteur.

de maison que formaient les rues Grange-Batelière, Pinon et Le Peletier. Sa façade principale donnerait sur cette dernière (ill. 3).

Après la détermination de la nature et de l'emplacement de la salle, il restait un dernier choix à effectuer, celui de l'architecte qui allait construire le nouvel Opéra. Même si la salle n'était que provisoire, il s'agissait d'un bâtiment public, royal et parisien, destiné à abriter une vénérable institution. La commande bénéficiait à ce titre d'un prestige considérable. Les candidats furent légion, à commencer par celui qui était perçu comme le plus légitime, Delannoy, qui veillait sur l'Opéra Richelieu depuis 1798. Au contraire de nombreux confrères qui se sont manifestés sur l'heure pour être chargés du chantier, François Debret (1777-1850) n'a pas soumis immédiatement de projet. Son statut d'architecte de la basilique SaintDenis aurait pu faire penser qu'il se tenait loin de la capitale et des rivalités d'architectes qui suivirent la fermeture de la salle Richelieu. Pourtant, il fut impliqué dès le départ dans le travail de réflexion et de prospection mené par l'administration pour trouver une solution à cette situation. Il visita au nom de la commission le Palais de la Bourse et la salle Favart en mars 1820, afin de déterminer si l'on pouvait y transférer l'Opéra. Lors de l'attribution du chantier de l'Opéra, fin juillet 1820, Debret avait comme avantages majeurs d'avoir été l'un des élèves de Fontaine, qui était membre de la commission, et surtout d'avoir été l'architecte chargé de la très récente restauration de l'Opéra de la rue Richelieu, en 1819. Sa connaissance parfaite de la salle à démonter et à reconstruire rue Le Peletier, ainsi que sa jeunesse (Delannoy avait alors soixante-cinq ans) jouèrent en sa faveur. La décision advint le 24 juillet 1820 . François Debret eut dès lors une dizaine de jours pour soumettre des plans et un devis. Sa proposition fut acceptée le 11 août, et il chiffra son devis à 872000 francs (ill. 4).

L'ordonnance royale du 9 août 1820 entérina les décisions liées à la conception de la salle et à sa localisation. Le roi y ordonna la construction d'une salle d'Opéra 


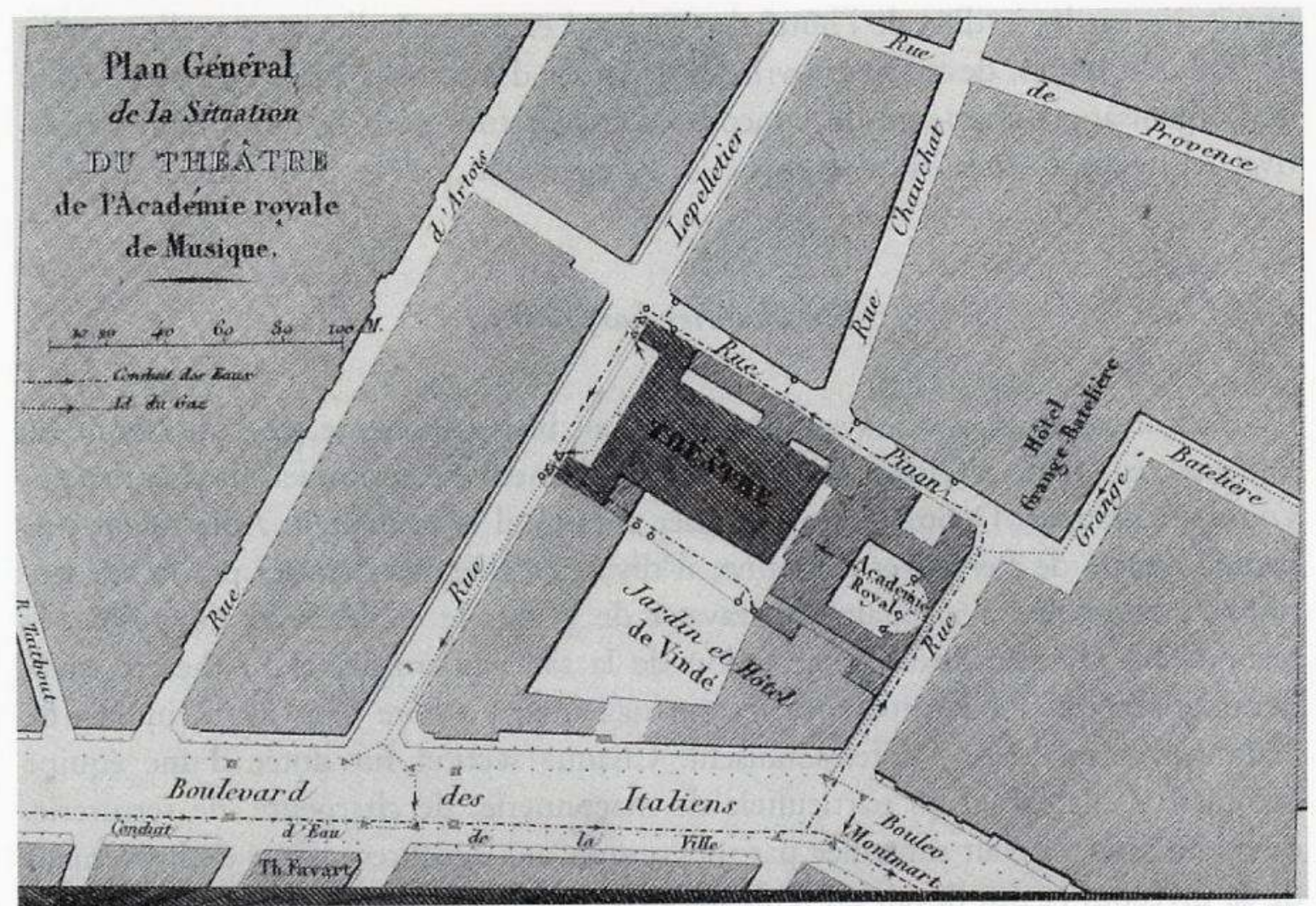

Ill. 3: Orgiazzi, Plan général de la situation du théatre de l'Académie royale de musique, détail de la planche 20 d'Architectonographie des théatres, 1837, Cabinet des arts graphiques du musée Carnavalet. Cl. de l'auteur.

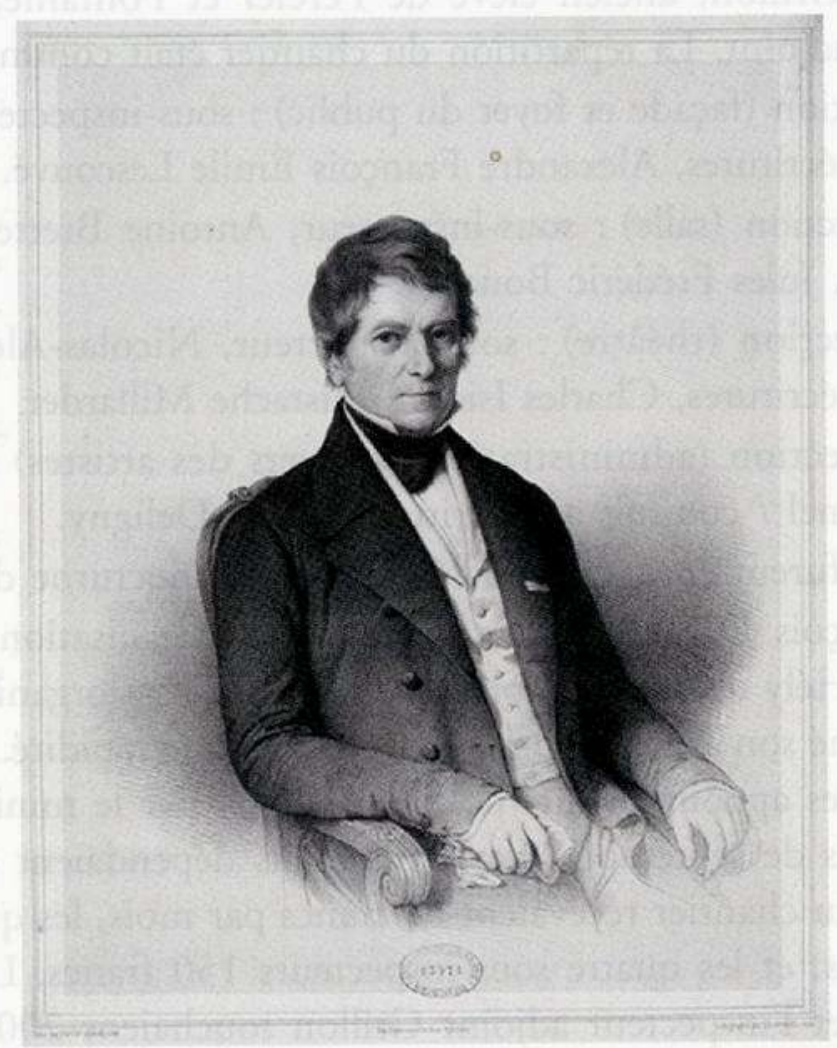

Ill. 4 : Fanny Alaux, Léon Noël, Portrait de François Debret, architecte du gouvernement, membre de l'Institut, 1850, lithographie, École nationale supérieure des Beaux-Arts. Cl. ENSBA. 
provisoire sur les jardins de l'hôtel de Choiseul et y associa l'ouverture d'un crédit de 900000 francs, destiné à couvrir les dépenses des travaux mais également celles de l'organisation ultérieure du concours d'architecture pour la future salle définitive. La livraison de la salle de la rue Le Peletier était prévue pour janvier 1821.

\section{Un chantier hors normes}

L'administration des Travaux de Paris était bien consciente, en la personne de son directeur Hély d'Oissel, du caractère absolument exceptionnel du chantier qui s'ouvrait. François Debret put s'organiser comme il le souhaitait. Après avoir pris conseil auprès de Percier et Fontaine, il divisa l'atelier du chantier en quatre sections: la première s'occupait des travaux de la façade et du foyer du public, la seconde fut chargée de la construction de la salle, la troisième du théâtre, et la dernière section devait superviser les aménagements à faire dans les bâtiments de l'administration, dans l'hôtel Choiseul. Chaque section fut dotée d'une équipe complète d'entrepreneurs particuliers de maçonnerie, de charpente, de serrurerie, de menuiserie, et était surveillée par un commis aux écritures chargé exclusivement de sa division, et qui devait en tenir les attachements en ordre et à jour. Un sousinspecteur fut également nommé par section. François Debret put choisir toute son équipe sauf l'inspecteur général du chantier, Guerchy, qui fut désigné par la commission. Tous les architectes employés étaient ou avaient été des élèves de François Debret. Grillon, ancien élève de Percier et Fontaine, puis de Debret, devint inspecteur adjoint. La répartition du chantier était comme suit :

- Première section (façade et foyer du public) : sous-inspecteur, Félix Duban / commis aux écritures, Alexandre François Émile Lescouvé.

- Deuxième section (salle) : sous-inspecteur, Antoine Brettenbauer / commis aux écritures, Jules Frédéric Bouchet.

- Troisième section (théâtre) : sous-inspecteur, Nicolas-Alexandre Dubois / commis aux écritures, Charles Isidore Eustache Millardet.

- Quatrième section (administration et foyers des artistes) : sous-inspecteur, Romain Fresnel / commis aux écritures, Louis Deligny.

Trois gardiens furent engagés pour la surveillance nocturne du chantier.

Bien sûr, François Debret dut soumettre cette organisation au directeur des Travaux de Paris. Hély d'Oissel l'approuva et le chantier s'organisa bien avant que le ministre ait donné son aval, toujours dans un souci de rapidité. L'état du personnel et la fixation des appointements ne furent signés par le ministre de l'intérieur qu'à la fin du mois de septembre. Les traitements dépendaient du statut de chacun : les gardiens du chantier recevaient 60 francs par mois, les quatre commis aux écritures 100 francs, et les quatre sous-inspecteurs 150 francs. Le conducteur des travaux, Boissard, et l'inspecteur adjoint Grillon touchaient 200 francs par mois. Le traitement de l'architecte et de l'inspecteur général répondait à une autre logique, plus complexe, héritée des lois impériales de 1808. Il se divisait entre une 
partie fixe et une partie proportionnelle aux dépenses du chantier. Leur salaire devait donc osciller entre 900 et 1000 francs mensuels.

Hély d'Oissel était tout à fait conscient que le personnel de l'agence était nombreux et qu'il allait coûter cher à l'administration. Mais il pensait cette organisation tout à fait légitime, étant donnés l'ampleur du chantier et le délai très court qui lui était accordé. Il le justifia avec force auprès du baron Mounier, le 14 août 1820 :

Si vous étiez tenté de trouver l'état-major un peu nombreux, je vous prierais [...] de remarquer que l'on demande ici à la Direction des travaux une sorte de tour de force, c'est-à-dire d'exécuter en trois ou quatre mois des travaux dont la dépense est présumée devoir s'élever à 830000 francs, qui dans les circonstances ordinaires ne s'exécuteraient qu'en deux ou trois années, que dès lors il est indispensable de dépenser en quelques mois ce qu'il en aurait coûté en deux ou trois années pour leur direction et leur surveillance 5 .

Ce découpage du chantier en quatre sections s'est avéré très efficace pour mener un chantier rapide, qui ne dura qu'un an. Debret, soutenu par sa hiérarchie et secondé par une équipe d'encadrement solide, parvint à diriger de nombreux entrepreneurs et plus de trois cents ouvriers pour bâtir une salle de spectacle de près de deux mille places en un temps record. En revanche, ce système n'a guère servi à maîtriser les dépenses, comme nous le verrons par la suite.

Le premier coup de pioche fut donné le 13 août 1820. Le duc de Choiseul, major général de la Garde nationale, habitait toujours l'hôtel particulier de la rue Grange-Batelière. Il accepta que les travaux commencent, mais pria que l'on veille à ce que les ouvriers ne s'approchent pas des appartements qu'il occupait ${ }^{6}$. Une palissade fut élevée à cet effet entre l'hôtel et les jardins. Debret perça une porte dans le mur de propriété sur la rue Le Peletier pour le service des premiers travaux. Le 16 août, Hély d'Oissel demanda au comte d'Anglès, préfet de Paris, d'interdire la circulation des cabriolets rue Le Peletier. Ce fut seulement le 13 septembre que la Garde nationale quitta définitivement l'hôtel de Choiseul. Selon Debret, ce délai d'un mois ralentit considérablement les travaux, en empêchant les sondages des murs de l'ancien hôtel particulier.

Le 29 septembre, la première pierre fut officiellement posée par le comte de Siméon, ministre de l'intérieur. La date n'était pas choisie au hasard : c'était le jour de la naissance du fils posthume du duc de Berry. D'une manière symbolique, le régime marquait dans la pierre la continuité de la royauté. François Debret avait dû concentrer ses efforts sur les fondations et des travaux annexes en attendant le terme de la grossesse de la duchesse. La pose de la première pierre le jour de la naissance du petit duc de Bordeaux ne donnait que plus de solennité à son œuvre, mais avait sans doute imposé un rythme inapproprié au début du chantier.

5. Hély d'Oissel au baron Mounier, 14 août 1820 , Arch. nat., $\mathrm{F}^{13} 700$.

6. Hély d'Oissel à Debret, 12 août 1820 , Arch. nat., $F^{21} 1273$. 
En novembre 1820, les murs en pierre de la façade du théâtre étaient déjà levés jusqu'au plancher du foyer du public. Ceux de la salle et du théatre (avant-scène, mur du lointain et murs latéraux) étaient également construits et attendaient de recevoir les pans de bois qui allaient les compléter. Ces derniers étaient en train d'être assemblés dans l'atelier de menuiserie. Quatre des six fermes des charpentes formant les combles de la salle étaient terminées et les menuisiers travaillaient activement sur les deux dernières. Pour le théâtre, six fermes sur sept étaient prêtes. L'atelier de menuiserie avait livré toutes les portes au peintre et se concentrait dorénavant sur les pilastres et les chapiteaux du grand foyer. Les trois rangs de devantures de loges étaient déjà terminés. Les murs du magasin du côté de la rue Pinon étaient montés à la hauteur de huit mètres et attendaient le levage de la charpente. Debret annonçait également que les logements de l'administration, les ateliers de tailleurs et les magasins d'habillement seraient prêts pour la fin du mois de novembre. Du côté des quartiers des artistes, vingt-huit loges étaient terminées et le premier plancher en pente du foyer de la danse était fini (ill. 5).

À l'arrivée de la mauvaise saison, le chantier semblait bien engagé. Toutes les parties de l'Académie royale de musique étaient bien avancées, en particulier le magasin de décors, qui n'attendait plus que sa charpente et sa couverture. En trois mois, l'architecte avait mené à bien la moitié du gros œuvre, fondations et rez-dechaussée. Restait alors à terminer les étages, à lever la charpente et à construire la couverture, alors que l'hiver arrivait. Pendant cette saison qui marque d'ordinaire un ralentissement dans le domaine du bâtiment, Debret maintint autant qu'il put une intense activité. Mais la rigueur de l'hiver 1820-1821 obligea les hommes à cesser le travail pendant près de quatre-vingt-dix jours cumulés ${ }^{7}$. Sur ce chantier, François Debret devait tout affronter à l'envers : les travaux extérieurs en hiver, et l'aménagement intérieur de la salle au retour des beaux jours.

Ces conditions difficiles participèrent à une hausse importante des coûts de construction, dont la main-d'œuvre, ainsi que l'évoqua François Debret :

Le levage de la charpente ayant été fait en hiver fut très dangereux pour les ouvriers qui en raison de cela exigèrent des prix plus élevés, sans quoi ils auraient abandonné leur travail ${ }^{8}$.

La direction des Travaux de Paris souligna les difficultés d'approvisionnement en matériaux, dues au peu d'élévation du niveau des cours d'eau lors de l'hiver 1820-1821. Par exemple, des conduites de gaz commandées aux usines du Creusot n'avaient pas pu être livrées par voie d'eau à cause du très bas niveau du canal du Centre et de la Loire. La livraison par la route, plus lente, augmenta de cent trente francs le coût de la tonne. Mais surtout, ce fut la concurrence d'autres chantiers dans Paris et dans les environs qui fit considérablement augmenter le coût du bois.

7. François Debret, Notice sur la construction de la salle provisoire de l'Académie royale de musique, 13 janvier 1833, Arch. nat., $\mathrm{F}^{21} 1581$.

8. Debret à Héricart de Thury, 21 février 1823, Arch. nat., $\mathrm{F}^{21} 1073$. 


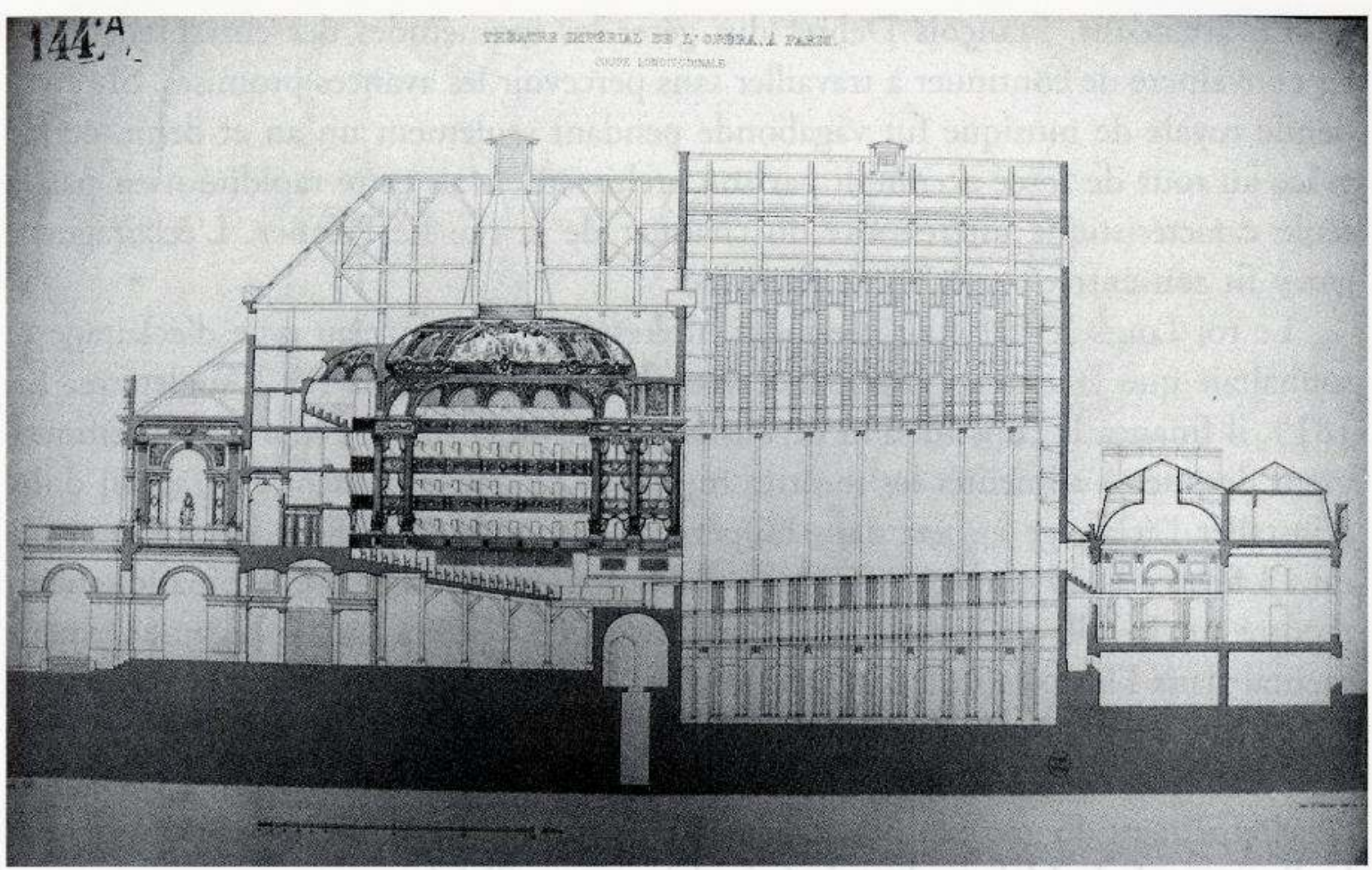

Ill. 5 : Clément Contant, Théatre impérial de l'Opéra à Paris. Coupe longitudinale, 1860, lithographie, Cabinet des arts graphiques du musée Carnavalet. Cl. de l'auteur.

Les magasins de Bercy, qui avaient brûlé peu de temps auparavant, étaient en train d'être reconstruits, mais surtout on bâtissait sur les boulevards deux autres théâtres (Les Panoramas et le Gymnase), soit deux constructions qui nécessitaient exactement les mêmes pièces de bois que l'Opéra. La nature du terrain provoqua également des dépenses inattendues. Des "constructions dans l'eau ", c'est-à-dire sur pilotis, s'avérèrent nécessaires : les fondations du théâtre du côté de la rue Pinon, ainsi que celles du pavillon faisant l'angle avec la rue Le Peletier devaient être construites sous le niveau des eaux.

Lors de la séance du 26 avril 1821, le ministre de l'intérieur Siméon présenta un projet de loi visant à faire passer le crédit alloué à la construction de la salle provisoire à 1800000 francs, soit le double de ce qui avait été prévu en août 1820 . En juin 1821, le roi ordonna l'ouverture d'un nouveau crédit de 300000 francs $^{10}$. Les parlementaires n'apprécièrent guère la multiplication des dépenses pour bâtir une salle provisoire. Comme le rappela justement Adolphe Jullien, François Debret travailla sous une pression constante :

En ces temps-là, les chambres n'étaient pas d'humeur accommodante sur le chapitre pécuniaire et l'on tracassa fort Debret pour ses devis dépassés ${ }^{11}$.

9. Debret à Héricart de Thury, 21 février 1823, Arch. nat., $\mathrm{F}^{21} 1073$.

10. Ordonnance royale du 20 juin 1821, Arch. nat., 1073.

11. Adolphe Jullien, Paris dilettante au commencement du siècle, p. 169. 
En attendant, François Debret dut gérer les inquiétudes des entrepreneurs et les convaincre de continuer à travailler sans percevoir les avances promises. Si l'Académie royale de musique fut vagabonde pendant seulement un an et demi, ce fut grâce au tour de force accompli par son architecte. Mais cette rapidité n'est pas la seule caractéristique intéressante du chantier de la rue Le Peletier. L'éclairage au gaz y fit son entrée sur la scène française.

Le roi Louis XVIII était vivement intéressé par ce nouveau type d'éclairage et souhaitait que la France rattrapât le retard qu'elle avait pris sur l'Angleterre. En 1819, il finança la construction de l'usine royale de la rue des Martyrs, spécifiquement destinée à alimenter les théâtres royaux ${ }^{12}$. Le gouvernement envisageait donc d'installer l'éclairage au gaz dans l'Opéra de la rue de Richelieu. Les événements du 13 février 1820 empêchèrent cette réalisation, et le projet fut automatiquement reporté sur la salle de Debret. La construction de l'Opéra fut un chantier expérimental dans l'histoire de l'éclairage au gaz, car il fut le premier exemple français d'installation de ce nouveau système dès la construction de l'édifice. Par son prestige, l'Opéra était considéré par le roi comme un modèle pouvant influer sur le développement du gaz dans les établissements privés. L'ambition royale, relayée par le directeur de la Maison du roi, était clairement affichée :

Je souhaite fort que l'éclairage par le gaz obtienne en France le même succès qu'en Angleterre, et cela dépend beaucoup de sa présence à l'Opéra ${ }^{13}$.

La fermeture précipitée de la salle Richelieu et la construction rapide de la salle Le Peletier prit de court les ingénieurs. Ils se retrouvèrent dans l'obligation de réfléchir à la conception d'un réseau de distribution, à la nature du gaz employé, à la construction d'un gazomètre et à une multitude de problèmes en même temps qu'avançait la construction de la salle. Ce n'est qu'en janvier 1821, soit cinq mois après le début des travaux, que le ministre de la maison du roi, le marquis de Lauriston, forma une commission afin de statuer sur ce nouveau type d'éclairage ${ }^{14}$, signe que ce nouvel équipement n'était pas encore rentré dans les mœurs. Six membres composèrent la commission : François Debret, Jean-Pierre Joseph Darcet et Charles Cagniard de la Tour, ingénieurs qui avaient réalisé l'installation du gaz à l'hôpital Saint-Louis, Pierre-Simon Girard, ingénieur supervisant la construction de l'usine royale, Nicolas-Alexandre Dubois, élève de Debret et chargé de faire un rapport sur les usines d'éclairage, et Antoine Courtin, administrateur du matériel de l'Académie de musique. Le baron de la Ferté présidait la commission, assisté de Gingembre, son adjoint. Cette commission eut une longévité exceptionnelle : elle

12. Lors de la fondation de la Compagnie royale du gaz en 1822, le ministre de la Maison du roi souligna les immenses avantages dont l'Angleterre avait bénéficié en appliquant la découverte française, et il se déclara " jaloux de rendre à son pays une industrie qui y avait pris naissance ". La Société royale du gaz fonctionna pendant dix ans, avant d'être rachetée à la révolution de 1830 par une compagnie anglaise, Manby et Wilson.

13. Pradel à La Ferté, 17 août 1820 , Arch. nat., $\mathrm{O}^{3} 1652$.

14. Lauriston à La Ferté, 17 janvier 1821, Arch. nat., $\mathrm{O}^{3} 1653$. 
se réunit pour la première fois le 7 février 1821 et n'arrêta ses travaux qu'en mars 1823. Elle ne tint pas moins de trente-trois séances. Sa durée montre les problèmes qui ont émaillé l'installation de l'éclairage par le gaz à l'Opéra Le Peletier : choix du type de gaz à utiliser, choix des conduites, choix des becs de gaz, construction du premier gazomètre parisien ${ }^{15}$, délimitation du réseau, etc. La commission répondit point par point à ces questions, et suivit de près leurs conséquences sur le terrain. Les travaux liés au gaz avançaient lentement car les ouvriers et les ingénieurs n'étaient pas au point vis-à-vis de cette industrie totalement nouvelle. L'usine royale fut prête à fournir du gaz le 30 avril 1821 au lieu du $1^{\text {er }}$ janvier, et le gazomètre de l'Opéra, situé dans un fond de parcelle rue Richer, présenta de nombreux problèmes de construction qui ne furent résolus qu'en 1823. La commission conseilla de ne pas appliquer ce nouveau système d'éclairage au théâtre, mais de le réserver à la salle, au foyer, aux corridors du public et au péristyle extérieur. Le procès-verbal de la séance ne mentionne pas les raisons qui ont motivé cette décision. Les membres de la commission ont sans doute craint d'appliquer à la zone la plus sensible de l'Opéra, l'espace scénique, une technologie sur laquelle on manquait encore de recul et d'expérience. L'Opéra Le Peletier se retrouva doté de près de 1200 becs de gaz. Les espaces réservés au public (façade, vestibule, salle, grands escaliers, foyer) en reçurent 900 et les espaces dédiés au fonctionnement interne du théatre (loges, bureaux de l'administration, foyer des artistes) n'en reçurent que 250. Les endroits dévolus à l'accueil du public sont logiquement les mieux pourvus : également plus spacieux et plus riches que les locaux de service, ils devaient donner une impression de magnificence et assurer la sûreté publique. Pour gagner du temps, la commission décida de confier la réalisation des becs de gaz à l'entrepreneur Creux. Cet industriel réputé avait déjà fourni l'hôpital SaintLouis et le palais du Luxembourg.

Le gaz arriva dans la nouvelle salle au milieu de la nuit du 20 juillet 1821 . Il pouvait dorénavant servir à éclairer d'une manière plus sûre les travaux dans les dessous du théâtre. Mais l'illumination complète de l'Académie ne pouvait encore se faire.

L'éclairage par le gaz de la façade, du lustre, de la salle, des corridors et de la rampe ne fut effectif qu'entre le 14 et le 16 août. Jusqu'à la dernière minute, rien n'était certain. Le fournisseur de becs Jecker fit un essai sur les lustres du grand foyer le 10 août. L'expérience fut un succès. Dans le cas contraire, on serait revenu à un éclairage à l'huile pour l'inauguration du 16, ce qui aurait été indigne de l'Opéra royal. Il fallait parer au plus pressé, aussi la commission établit comme priorité l'illumination de la façade, du foyer, des corridors du public, de la rampe et de la salle :

En ce qui concerne l'éclairage des façades latérales et lieux autres que ceux désignés ci-dessus, cet éclairage sera provisoirement établi à l'huile, et l'éclai-

15. Germain Bapst, Essai sur l'histoire du théatre, p.539. 
rage par le gaz n'y sera introduit et adapté que lorsque l'on sera sorti des embarras et des difficultés de l'ouverture du théâtre ${ }^{16}$.

Le provisoire fut dans certains cas destiné à durer : les loges des artistes et les dépendances de certaines grandes loges fonctionnaient encore à l'huile dans les années 1850.

Les tâtonnements de l'administration concernant l'installation de l'éclairage au gaz à l'Opéra ralentirent l'ensemble de l'aménagement intérieur, mais permirent aux spectateurs de découvrir la nouvelle salle à la douce lueur des becs de gaz.

\section{Livraison de la salle et inauguration}

Sous-estimée dès l'ordonnance du 9 août 1820 , la durée de construction de la salle Le Peletier prit douze mois au lieu de quatre. Son ouverture, annoncée pour janvier, repoussée à juillet, n'eut finalement lieu que le mois suivant.

L'Opéra de la rue Le Peletier fut inauguré le 16 août 1821, en présence des ministres de l'intérieur et de la maison du roi, d'Hély d'Oissel et de François Debret et son équipe. Pour l'occasion, la troupe de l'Académie royale donna l'opéra Les Bayadères de Jouy et Catel, et le ballet Le Retour de Zéphire de Gardel et Steibelt. Ces deux œuuvres avait déjà été jouées à Paris ${ }^{17}$, y avaient connu un grand succès, mais manquaient d'originalité pour une inauguration. L'ambiance semblait assez maussade, et les artistes ne se montrèrent pas à leur meilleur niveau. Madame Le Branchu, première chanteuse, était indisposée mais se produisit tout de même: elle "toussa son rôle plutôt qu'elle ne le chanta " ${ }^{18}$. Étrangement, le public ne s'est pas pressé pour la cérémonie d'inauguration de l'Opéra provisoire. Les loges étaient combles, mais les banquettes du parterre ne firent pas le plein : des places restèrent libres jusqu'à la moitié de la représentation. La recette ne se chiffra qu'à 9 251,10 francs ${ }^{19}$, ce qui était honorable, sans plus.

La raison de ce semi-échec tient à la concurrence exceptionnelle qu'il y avait ce soir-là dans Paris : on jouait quatre premières de spectacles, en plus de l'inauguration du nouvel Opéra. La Gaîté présenta les Époux de quinze ans de Paul, le Vaudeville Le Traité de Paix de Dartois et Brizé. Le Gymnase donna la première du Mariage Enfantin de Scribe, et le public pouvait enfin découvrir Le Philosophe en Voyage à l'Opéra-Comique, sur un livret du célèbre Paul de Kock et une musique de Kreubé et Pradher. Ces théâtres firent tous salle comble. La concentration de nouveautés dans la même soirée était inédite, et ne pouvait être un hasard de la programmation. Ces théâtres voyaient-ils dans cette sorte de sabotage de la

16. Procès-verbal de la commission, séance du 28 juillet 1821 , Arch. nat., $\mathrm{O}^{3} 1654$.

17. Elles ont été créées respectivement en 1810 et 1802.

18. Albert de Lasalle, Les treize salles de l'Opéra, p. 239.

19. Adolphe Jullien, Inauguration de la salle de l'Opéra, le 16 août 1821. 


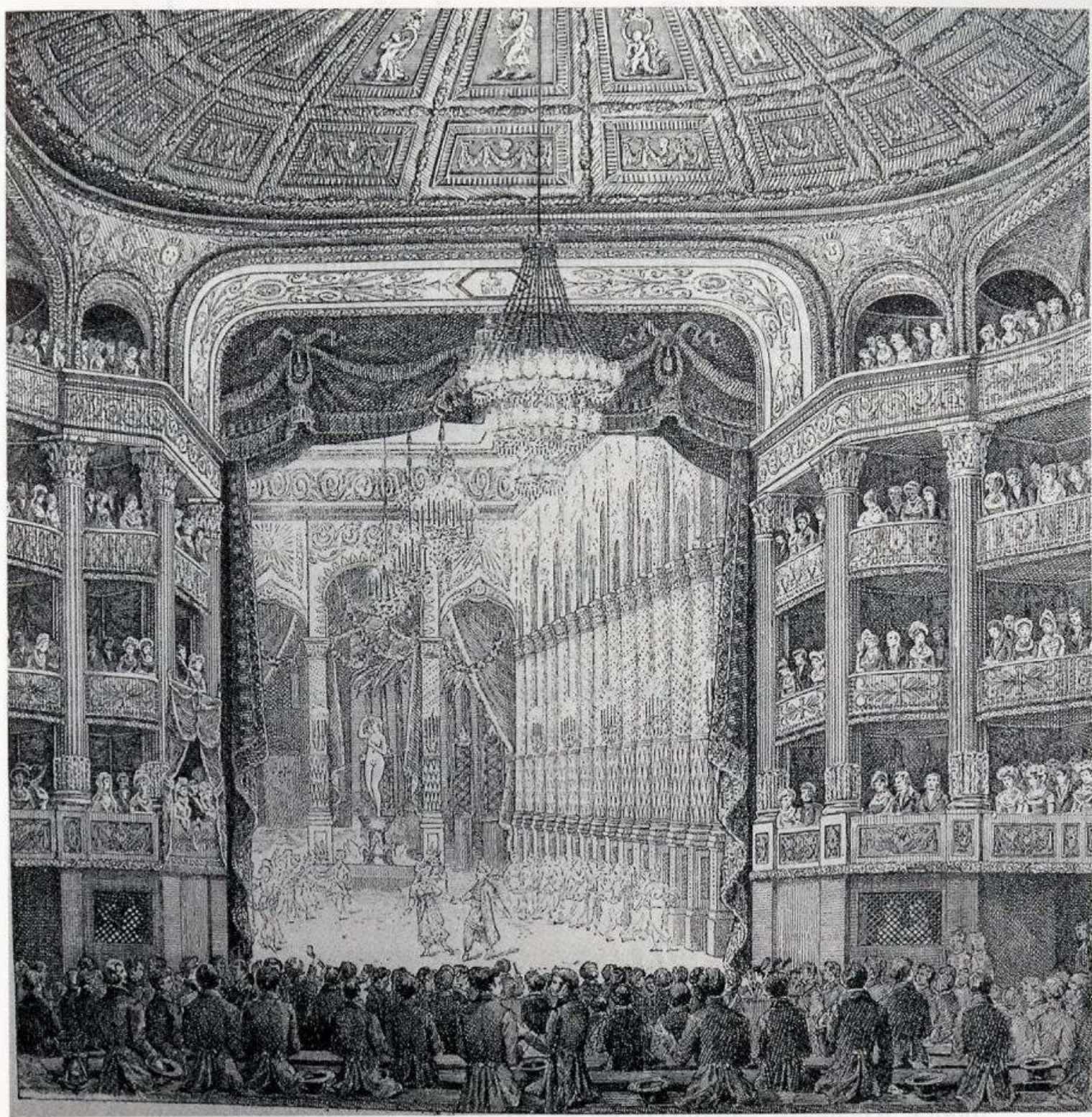

Ill. 6: B. Drouot, Représentation des Bayadères pour l'inauguration de l'Opéra de la rue Le Peletier, le 16 août 1821, [1821], lithographie, Cabinet des arts graphiques du musée Carnavalet. Cl. de l'auteur.

soirée inaugurale de l'Opéra une occasion de protester contre la redevance qu'ils devaient lui payer tous les ans ? Le doute est peu permis face à une telle concentration de nouveautés à la date bien précise du 16 août. Par ailleurs, j'ignore si le roi Louis XVIII et les membres de la famille royale ont assisté à l'inauguration de la salle. Ils ne sont pas mentionnés dans les textes et dans les archives, bien qu'ils figurent dans un tableau censé représenter l'inauguration de la salle de Debret. Sans doute ont-ils préféré ne pas parâtre lors d'une festivité dans l'enceinte de l'institution où avait été assassiné l'un des leurs, tout comme ils n'avaient pas posé la première pierre de l'édifice. L'absence du faste entourant le roi et sa cour est peut-être une raison supplémentaire expliquant que les parisiens aient préféré, ce soir-là, la nouveauté d'une pièce de théâtre à la nouveauté toute relative d'une salle provisoire (ill. 6). 
La presse eut du mal à s'accorder sur la valeur de la représentation qui s'était déroulée ce soir-là, et encore plus sur le crédit à accorder à cette nouvelle salle de spectacle. Tous les journalistes louèrent la beauté du foyer du public et celle de la salle, mais cette dernière, en majorité héritée de la rue de Richelieu, n'était guère une nouveauté. Le Journal des dames et des modes fut ravi de la soirée et du nouvel Opéra, dont il fut d'ailleurs le seul à fournir une description à ses lectrices. Il renseigna sur l'ambiance festive qui régnait autour du monument pendant la soirée : de nombreux promeneurs conversaient avec des badauds assis admirant le défilé des voitures, les fenêtres de la rue Le Peletier et des rues alentours étaient noires de monde. L'organe officiel, le Moniteur Universel, remplit son rôle à merveille : il déclara que la salle était une réussite et que les artistes avaient été éclatants. Il félicita l'administration de l'Opéra de n'avoir pas choisi de présenter une nouveauté ce soir-là. Car :

On n'est pas venu pour [les artistes], on est venu pour voir la nouvelle salle, et pour s'y faire voir ${ }^{20}$.

Le Journal de Paris parut également satisfait de cette nouvelle salle. Il remarqua tout de même que l'effet général de la représentation avait été froid et dépourvu de génie. Ce bémol fut également souligné par le Miroir, organe de presse des libéraux. Ces journalistes de l'opposition furent assez honnêtes pour reconnaître les qualités de l'édifice, et, non sans allusions subtiles au nouvel ordre politique qu'ils désiraient voir s'installer dans la société, ne firent que des éloges à l'Opéra provisoire :

Les piétons savent gré à $\mathrm{M}$. Debret de toutes les précautions qu'on a prises pour l'entrée et la sortie, des issues ménagées avec art et des abris préparés avec soin ; les personnes du parterre, toutes surprises d'avoir des places commodes, félicitaient l'architecte de ne point avoir oublié qu'à l'époque où nous vivons, il ne suffit plus de satisfaire les premières loges; l'opinion du parterre a bien autant d'importance.

De leur côté, les âmes chagrines rivalisèrent de mauvaise volonté. Le journaliste du Drapeau blanc, lassé d'avance de toutes les pièces présentées à Paris ce soir là, écrivit que finalement, sur cinq nouveautés, rien n'était bien nouveau, pas même la salle de l'Académie royale de Musique. Le chroniqueur des Débats trouva tout mauvais : l'opéra joué ne lui convenait pas, les chanteurs étaient enroués, les sièges mauvais, le vernis tacha ses vêtements et dégageait une odeur entêtante, insupportable, et l'éclairage de la salle était mal adapté. Le journaliste de la Quotidienne fit mieux : il claqua la porte de l'Opéra dès la fin du premier acte des Bayadères, disant ne plus supporter de voir à nouveau cet opéra. Il se contenta donc de rapporter les

20. Toutes les citations qui suivent sont extraites d'Adolphe Jullien, Paris dilettante au commencement du siècle, Paris, Firmin-Didot, 1884. 
plus mauvais échos recueillis auprès de ses confrères à la sortie de la représentation. Le chroniqueur de la Gazette de France se joignit au concert de critiques : tout lui sembla épouvantable, surtout la visibilité. Ainsi écrivit-il le lendemain :

Un cri général qui s'est élevé de toutes les loges de côtés atteste que, sur dix personnes qu'elles contiennent, QUATRE sont condamnées à s'en rapporter au récit des autres.

La mauvaise visibilité des loges de côté n'était pourtant pas chose nouvelle. Évariste Dumoulin, chroniqueur au Constitutionnel, fut peut-être le plus sévère. Il reprocha à Debret de n'avoir pas tenu compte de la situation engoncée du théâtre pour lui donner une élévation adaptée. Sa façade aurait dû être conçue pour être vue de près. Ne l'étant pas, elle apparaissait "bizarre et mesquine " et l'imitation de Palladio était complètement ratée : l'architecte italien était traité dans l'œuvre de Debret comme "un géant sous l'habit d'un nain " ${ }^{21}$. Enfin, le journaliste ajouta qu'il craignait fort que l'architecte n'eût pas respecté les règles de l'acoustique.

En plus d'avoir été inauguré sans grand panache, le nouvel édifice a, semblet-il, été mal accueilli par la population parisienne. Elle lui trouva une situation peu avantageuse, coincée dans les édifices alentours et sans dégagement. La façade fut jugée sans caractère et surtout d'une simplicité effrayante. Certains le comparèrent avec un restaurant de la place du Châtelet, "Au Veau qui tète ". Si cette comparaison ironique peut en partie se comprendre - les deux édifices présentant un premier étage percé d'une enfilade de baies cintrées - elle restait cruelle quant à l'appellation du restaurant, qui, appliquée à l'Opéra, ridiculisait impitoyablement ce haut lieu de l'art. Pour d'autres, le Grand Opéra ne se distinguait en rien des autres immeubles de la rue Le Peletier. Ainsi, quand un étranger demandait à un parisien où se situait l'Opéra, ce dernier lui répondait " rue Le Peletier, la deuxième porte cochère à main droite ${ }^{22}$ ". La simplicité de la façade fut en outre rapidement associée à celle supposée de son architecte. Les chroniqueurs de l'époque et les historiens répétèrent à l'envi ce bon mot, qui circulait, paraitt-il, de bouche en bouche quand on passait devant la façade de l'Académie Royale de Musique :

Tiens, il n'y a que huit muses sur cette façade, quelle est celle qui manque ?

- Vous ne voyez pas que c'est celle de l'architecture ${ }^{23}$ !

Cette épigramme pourrait fonctionner si seulement il existait une muse de l'architecture. Mais si, comme l'affirme Jacques Hillairet dans le Dictionnaire historique des rues de Paris $^{24}$, la muse manquante était réellement celle de la Musique, cette absence sur le fronton de l'Académie royale de musique était sans doute pire que celle d'une éventuelle muse de l'architecture (ill. 7 et 8).

21. Lettre à Madame de B... sur la nouvelle salle d'Opéra, précédé de stances à F. Debret, p. 19.

22. Cité par Louis Lurine dans Les rues de Paris. Paris ancien et moderne, p. 373.

23. Albert de Lasalle, Les treize salles de l'Opéra, Sartorius, Paris, p. 170.

24. Jacques Hillairet, Dictionnaire historique des rues de Paris, article "Le Peletier ", t. 2, p. 37-38. 


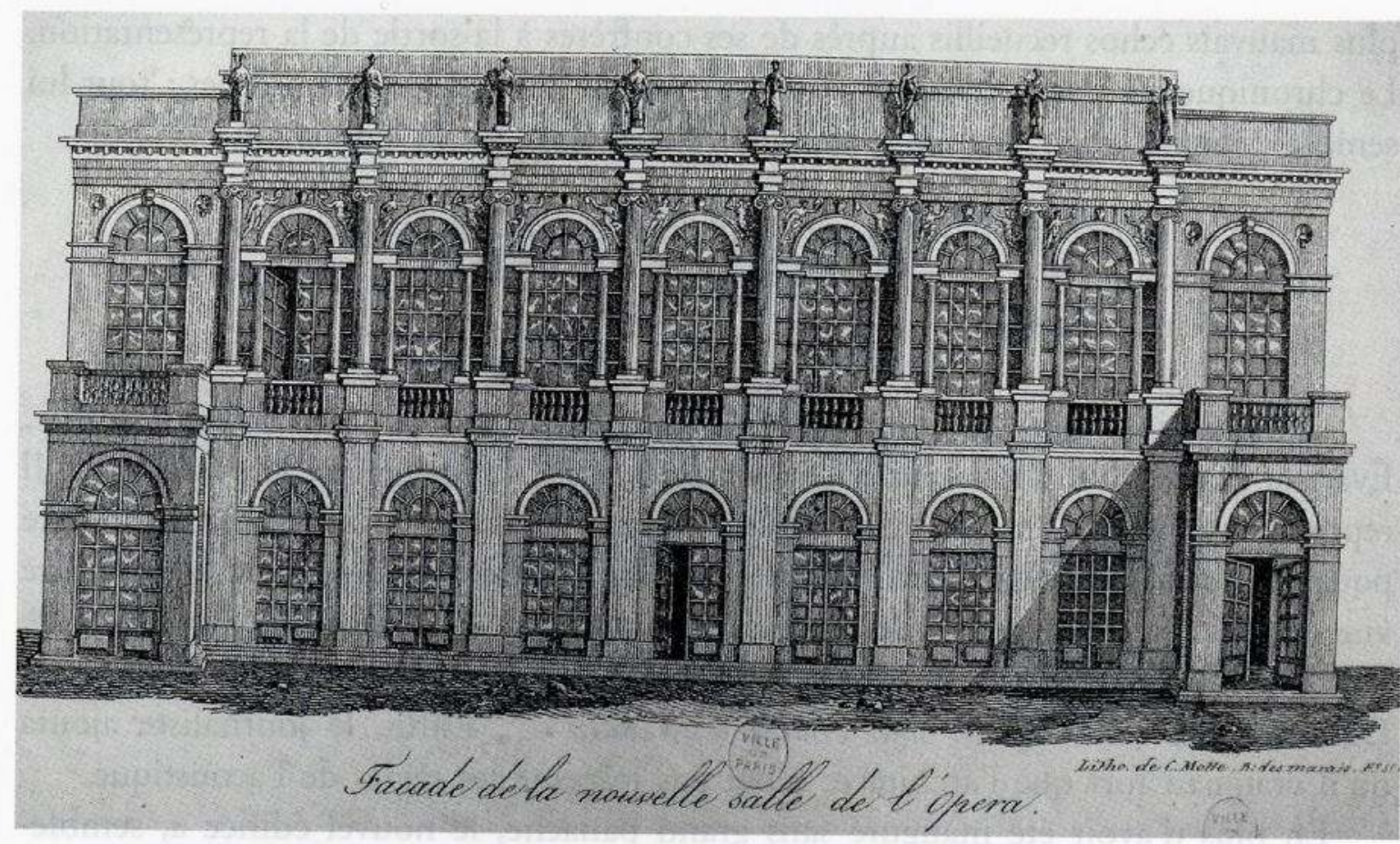

Ill. 7 : C. Motte, Façade de la nouvelle salle de l'Opéra, [v. 1821], lithographie, Cabinet des arts graphiques du musée Carnavalet. Cl. de l'auteur.

Malgré des débuts quelque peu mouvementés, la salle de Debret fit bientôt sa place dans l'espace culturel et mondain parisien. La puissance de sa machinerie, la commodité de ses espaces publics, la beauté et l'acoustique de sa salle en firent un haut lieu de la musique européenne. Les plus grands vinrent s'y produire, et, cette salle censée ne durer que dix ans maximum s'installa durablement dans le paysage de la capitale. Bientôt flanquée de galeries marchandes lui offrant une ouverture sur le boulevard des Italiens, fréquemment restaurée et bénéficiant rapidement des dernières inventions (électricité, bitume), la salle Le Peletier réussit à pallier les défauts de sa construction légère. L'édification d'une salle définitive, véritable serpent de mer dans l'administration, était régulièrement évoquée, mais aucun gouvernement ne s'y attela. On se satisfit ainsi de la salle provisoire de Debret jusqu'à ce qu'Orsini attente à la vie de Napoléon III le 14 janvier 1858 devant l'Opéra. Une nouvelle fois, ce fut un attentat contre une personne royale qui décida de l'avenir de l'Académie de musique. Le concours pour une salle définitive, mis en attente depuis 1820, fut lancé en 1859. Il aboutit à la victoire de Charles Garnier, dont l'Opéra inauguré en 1875 allait plonger dans l'oubli son prédécesseur, certes plus modeste mais tout aussi intéressant pour l'histoire de l'architecture théâtrale française.

Elsa CHerbuy

diplômée de master 2 à l'Éphé, archiviste paléographe 


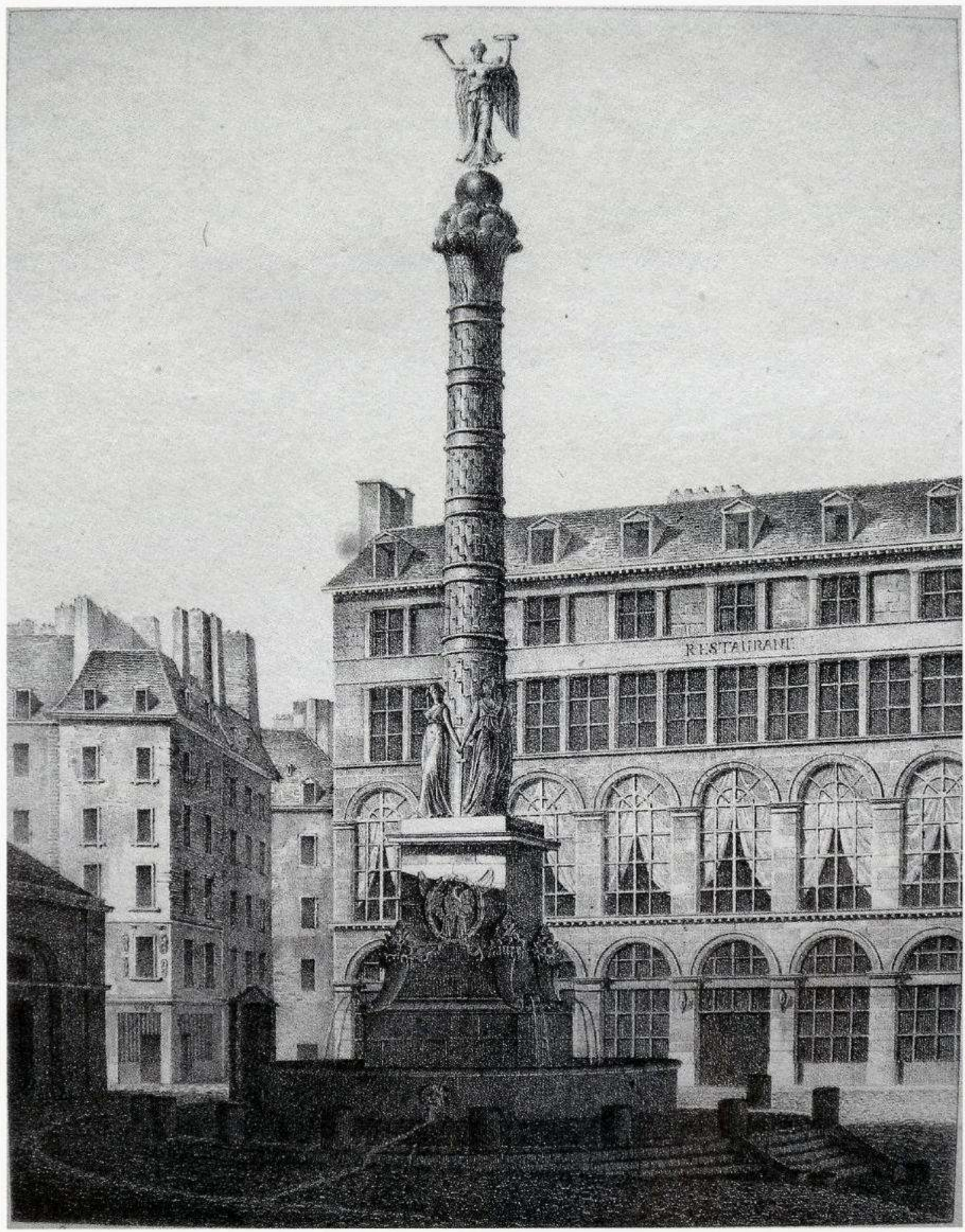

Ill. 8: Jean-Baptiste Arnoult, François-Séraphin Delpech, Vue de la fontaine de la place du Châtelet, 1820, lithographie. Arch. nat., fonds Debret 568AP. Cl. de l'auteur. 